\title{
Genomic Evidence of Gene Flow During Reinforcement in Texas Phlox
}

\section{Citation}

Roda, Federico, Fábio K. Mendes, Matthew W. Hahn, and Robin Hopkins. 2017. Genomic Evidence of Gene Flow During Reinforcement in Texas Phlox. Molecular Ecology 26, no. 8: 2317-2330.

\section{Permanent link}

http://nrs.harvard.edu/urn-3:HUL.InstRepos:41302400

\section{Terms of Use}

This article was downloaded from Harvard University's DASH repository, WARNING: This file should NOT have been available for downloading from Harvard University's DASH repository.

\section{Share Your Story}

The Harvard community has made this article openly available. Please share how this access benefits you. Submit a story.

\section{Accessibility}




\title{
Genomic evidence of gene flow during reinforcement in Texas Phlox
}

\author{
FEDERICO RODA,* (iD FÁBIO K. MENDES, $\uparrow$ MATTHEW W. HAHN $\dagger+$ and ROBIN HOPKINS* \\ *Department of Organismic and Evolutionary Biology, The Arnold Arboretum of Harvard University, 1300 Centre Street, \\ Boston, MA 02131, USA, †Department of Biology, Indiana University, 1001 E. Third Street, Bloomington, IN 47405, USA, \\ $\$$ School of Informatics and Computing, Indiana University, 1001 E. Third Street, Bloomington, IN 47405, USA
}

\begin{abstract}
Gene flow can impede the evolution of reproductive isolating barriers between species. Reinforcement is the process by which prezygotic reproductive isolation evolves in sympatry due to selection to decrease costly hybridization. It is known that reinforcement can be prevented by too much gene flow, but we still do not know how often have prezygotic barriers evolved in the presence of gene flow or how much gene flow can occur during reinforcement. Flower colour divergence in the native Texas wildflower, Phlox drummondii, is one of the best-studied cases of reinforcement. Here we use genomic analyses to infer gene flow between $P$. drummondii and a closely related sympatric species, Phlox cuspidata. We de novo assemble transcriptomes of four Phlox species to determine the phylogenetic relationships between these species and find extensive discordance among gene tree topologies across genes. We find evidence of introgression between sympatric $P$. drummondii and $P$. cuspidata using the $D$-statistic, and use phylogenetic analyses to infer the predominant direction of introgression. We investigate geographic variation in gene flow by comparing the relative divergence of genes displaying discordant gene trees between an allopatric and sympatric sample. These analyses support the hypothesis that sympatric $P$. drummondii has experienced gene flow with P. cuspidata. We find that gene flow between these species is asymmetrical, which could explain why reinforcement caused divergence in only one of the sympatric species. Given the previous research in this system, we suggest strong selection can explain how reinforcement successfully evolved in this system despite gene flow in sympatry.
\end{abstract}

Keywords: introgression, Phlox, phylogenetic network, reinforcement, transcriptome

Received 8 September 2016; revision received 17 January 2017; accepted 18 January 2017

\section{Introduction}

An ever-increasing number of genomic studies are revealing a history of gene flow between closely related lineages (Mallet et al. 2016). The prevalence of hybridization between taxa enlivens interest in how barriers to reproduction evolve in the presence of gene flow. Much research has focused specifically on understanding the role of gene flow during reinforcement. Reinforcement is the process through which prezygotic reproductive isolation evolves in response to selection

Correspondence: Federico Roda, Fax: 617384 6596;

E-mail: federicoroda@fas.harvard.edu against costly hybridization (Butlin 1987; Hopkins 2013; Liou \& Price 1994; Servedio \& Noor 2003). Reinforcement occurs when diverged taxa hybridize and selection against inviable, maladapted or sterile hybrids favours traits that increase prezygotic reproductive isolation (Butlin 1987; Hopkins 2013; Liou \& Price 1994; Servedio \& Noor 2003), leading to the evolution of assortative mating in sympatric populations.

Dozens of empirical studies suggest the importance of reinforcement during speciation (e.g. Albert \& Schluter 2004; Ortiz-Barrientos et al. 2004; Hoskin et al. 2005; Matute 2010; Hopkins \& Rausher 2012), yet there are still outstanding questions as to how this process occurs in the presence of gene flow between 
hybridizing taxa. Extensive theoretical research has investigated how gene flow can influence the successful evolution of reproductive isolation in sympatry (e.g. Barton \& De Cara 2009; Felsenstein 1981; Kirkpatrick \& Servedio 1999; Liou \& Price 1994; Liou \& Price 1994; Sanderson 1989; Servedio \& Kirkpatrick 1997; Servedio \& Noor 2003), but there are few empirical studies validating this theory (but see Matute 2010).

Hybridization is a double-edged sword during the process of reinforcement. Costly hybrid matings generate the selective force favouring the increase in reproductive isolation, yet hybridization with gene flow can erode species differences and prevent the fixation of new barriers to reproduction (Bank et al. 2012; Kirkpatrick 2000; Liou \& Price 1994). As species diverge, gene flow with recombination can break up the associations between co-adapted traits that define each lineage and can prevent the evolution of new traits that could cause reproductive isolation (Kirkpatrick 2000; Liou \& Price 1994; Nosil 2013; Nosil et al. 2003; Yukilevich 2012). It has therefore been argued that the evolution of increased prezygotic reproductive isolation in sympatry occurs with little or no gene flow (Felsenstein 1981; Butlin 1987). Yet, theoretical research has shown that, under certain evolutionary conditions, reinforcement can be successful in the face of gene flow (Servedio \& Kirkpatrick 1997; Kirkpatrick \& Servedio 1999; Kirkpatrick \& Ravigne 2002; Barton \& De Cara 2009).

Empirical evidence that reinforcement does occur in the face of gene flow is limited. An experimental evolution study in Drosophila demonstrated that reinforcement can occur despite some gene flow between lineages experiencing strong selection against hybrids (Matute 2010). Furthermore, genomic analyses have revealed a history of gene flow between Drosophila pseudoobscura and Drosophila persimilis (Kulathinal et al. 2009), which have undergone reinforcement for assortative mating in sympatry (Noor 1995). In other examples of divergence likely caused by reinforcement, postzygotic reproductive isolation between sympatric lineages is not complete, creating the potential for gene flow (Gerhardt 1994; Höbel \& Gerhardt 2003; Smadja \& Ganem 2005; Smadja et al. 2015). More research is needed to determine if and how gene flow occurs during the process of reinforcement.

A well-characterized example of reinforcement is flower colour divergence in Texas wildflowers in the Phlox genus (Levin 1985; Hopkins \& Rausher 2011, 2012, 2014; Hopkins et al. 2012, 2014). Phlox drummondii, Phlox cuspidata and Phlox roemeriana make up a monophyletic clade of annual Phlox species that grow in Texas (Ferguson et al. 1999a; Ferguson \& Jansen 2002). All three species share a similar light-blue flower colour throughout most of their ranges, but where
P. drummondii and P. cuspidata co-occur in sympatry, P. drummondii has dark-red flower colour (Fig. 1a). The evolution of flower colour in sympatry decreases hybridization by as much as 50\% (Hopkins \& Rausher 2012). Despite the reduction in hybridization, hybrids can be found in nature (Levin 1967; Ferguson et al. 1999b; Ruane \& Donohue 2008; Ruane 2009). Low fertility in hybrids causes selection against hybridization and favours flower colour divergence (Hopkins et al. 2012).

(a)
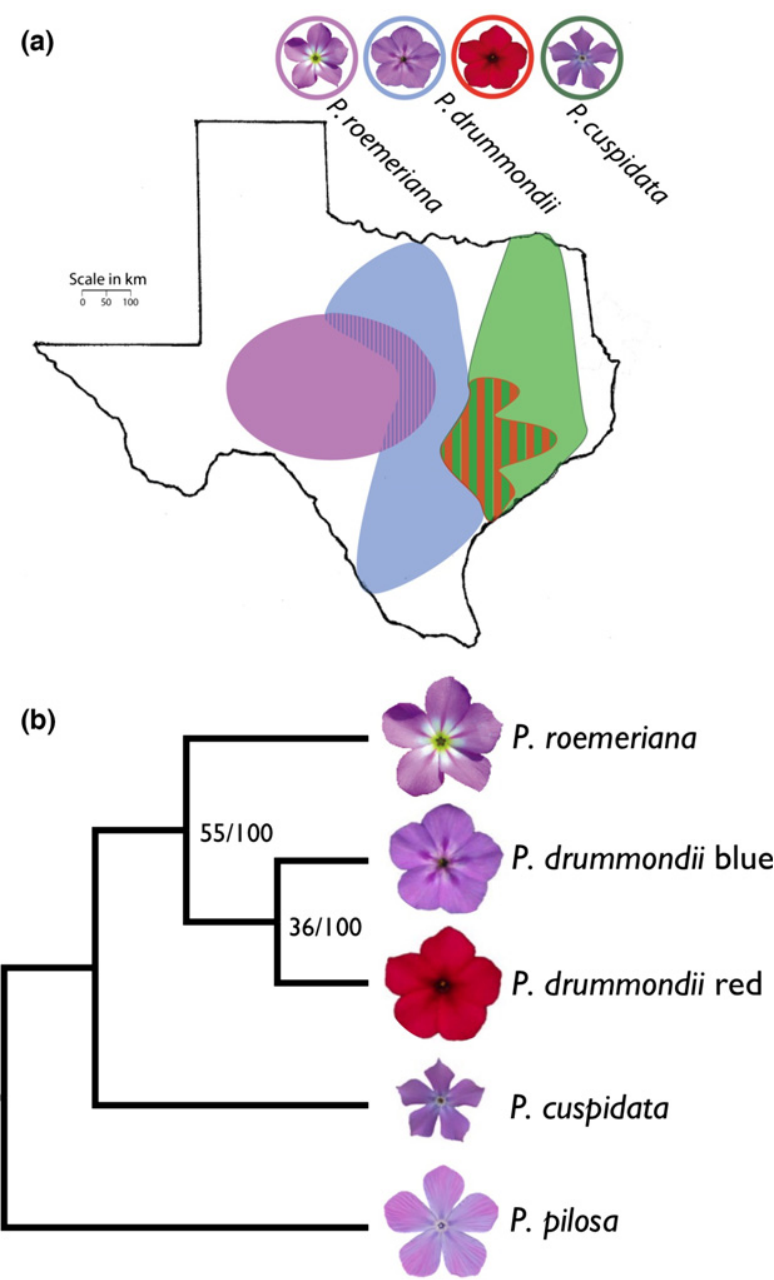

Fig. 1 Distribution and phylogenetic relationships of Texas Phlox. (a) Schematic of Texas Phlox ranges with representative flowers of each species. Coloured circles around flowers correspond to range colour on map. (b) Cladogram summarizing results from phylogenetic analyses of Texas annual Phlox transcriptomes. Phlox pilosa was used as outgroup. Analyses of concatenated sequences and gene trees converged on the same topology. On the right of tree nodes, we show concordance factors from the consensus tree, and bootstrap support from the parsimony-based analysis, respectively. Analyses of the concatenated alignment resulted in fully supported phylogenies, while analyses employing gene tree topologies had lower support (see Table S1, Supporting information). 
Yet this hybrid sterility could also prevent gene flow between these two Phlox species. It is unclear based on field observations if gene flow occurs (or has occurred) between these species.

Recent advancements in methods to detect introgression using genomic data offer a new opportunity to understand the process of reinforcement (Holder et al. 2001; Joly et al. 2009; Martin et al. 2015; Rosenzweig et al. 2016). Here, we employ transcriptome data from $P$. drummondii and P. cuspidata in three complementary strategies to detect the presence and direction of gene flow during reinforcement. First, we investigate patterns of divergence at multiple informative sites using the $D$ statistic [otherwise known as the 'ABBA-BABA' test (Green et al. 2010)]. Second, we use gene trees to estimate phylogenetic networks-which include reticulate events such as hybridization (Solís-Lemus \& Ané 2016) -and to estimate the amount of gene flow between lineages (Gronau et al. 2011). Finally, we investigate geographic variation in gene flow by comparing the relative divergence of genes with discordant gene trees across multiple samples. Our results suggest that P. drummondii and P.cuspidata exchanged genes recently, in the presence of strong reinforcing selection.

\section{Methods}

Below we describe our strategy for sequencing and assembling de novo transcriptomes from five Phlox individuals and discuss our analyses for inferring introgression and divergence using these data. For readability, we have listed the specific parameter values for each software package in Table S1 (Supporting information).

\section{Transcriptome assembly and orthology inference}

RNA extraction and sequencing. We sequenced the transcriptomes of four Phlox species growing natively in Texas: Phlox drummondii with dark-red flowers (hereafter named DruR), Phlox cuspidata (Cusp), Phlox roemeriana (Roem) and Phlox pilosa (Pilo, Fig. 1a, Table S2, Supporting information). To avoid biased estimates of introgression, we selected plants that phenotypically do not look like hybrids and are from populations that include no visible hybrids. Early-generation hybrids can be easily distinguished on the basis of their reproductive and vegetative morphologies (Levin 1975). We sampled five tissues from one individual of each species: flower bud, mature flower, leaf, stem and root. RNA was extracted using E.Z.N.A. ${ }^{\circledR}$ Plant RNA Kit (Omega Bio-tek ${ }^{\circledR}$ ), including three additional washes with $70 \%$ ethanol before membrane elution. Equimolar amounts of RNA from the different tissues of an individual were pooled. The four pools were prepared using Apollo Poly-A isolation and mRNA directional library preparation, and then, $2 \times 150$-bp paired-end sequences were obtained using an Illumina HiSeq 2500. Sequencing and library preparation was conducted at the Bauer Core of Harvard University.

Additionally, we sequenced the transcriptome of a second $P$. drummondii individual with light-blue flower colour (named DruB). For the remainder of this study, we refer to the two P. drummondii samples by their colour (i.e. light blue and dark red). This individual had only three of its tissues sampled (flower, bud, leaf); here, RNA was extracted using the Spectrum ${ }^{\mathrm{TM}}$ Plant Total RNA Kit from Sigma Aldrich, and mRNA isolated for sequencing using a polyA Spin ${ }^{\mathrm{TM}}$ Kit. For this sample, $2 \times 125$-bp paired-end reads were sequenced on an Illumina HiSeq 2500 at the ICMB Genomic Sequencing and Analysis Facility of The University of Texas at Austin.

Transcriptome assembly and orthology inference. We used Trimmomatic (Bolger et al. 2014) to filter low-quality paired-end sequence reads, and Trinity (Grabherr et al. 2011; Haas et al. 2013) to de novo assemble the transcriptome of each of the five individuals. Assembly statistics are provided in Table 1. We identified coding sequences (CDS) using TRANSDECODER (Haas et al. 2013), and reduced sequence redundancy with CD-HIT-EST (Li \& Godzik 2006).

We used the tree-based ortholog identification pipeline described in Yang \& Smith (2014) across our five samples. In short, we used all-by-all BLASTN (Altschul

Table 1 Summary of transcriptome assemblies

\begin{tabular}{lrlrlrr}
\hline Taxa & Number genes & Isoforms/gene & N50 length & Median length & Average length & Assembled bases \\
\hline Phlox cuspidata & 104573 & 1.44 & 612 & 342 & 503.74 & 76081919 \\
Phlox drummondii light-blue & 99962 & 1.74 & 1312 & 698 & 903.84 & 157040540 \\
P. drummondii dark red & 72749 & 1.48 & 592 & 339 & 489.62 & 52718969 \\
Phlox pilosa & 75304 & 1.39 & 515 & 321 & 453.39 & 47571874 \\
Phlox roemeriana & 81709 & 1.63 & 778 & 374 & 574.13 & 76289085 \\
\hline
\end{tabular}


et al. 1997) and MCL (Enright et al. 2002; Van Dongen \& Abreu-Goodger 2012) on all nonredundant CDS sequences to obtain clusters of similar sequences, which were then aligned with MAFFT (Katoh \& Standley 2013), and trimmed with Phyutility (Smith \& Dunn 2008). Initial phylogenetic trees were then estimated for each cluster using fastTReE (Price et al. 2010). Because these trees may contain long branches resulting from misassembly, recombination or paralogy, we trimmed branches more than 10 times longer than the average distance to tips seen in its sister clade, and longer than 0.4 substitutions per site. Sequences from each subtree were realigned using MAFFT, and gene trees were reinferred using RAXML (Stamatakis 2006) and submitted to one last removal of long branches.

We further pruned the homologous gene trees to a single orthologous sequence per sample using the maximum inclusion (MI) method (Dunn et al. 2008, 2013; Smith et al. 2011; Yang \& Smith 2014). This method involves isolating the subtree with the highest number of taxa without taxon duplication. The resulting trees were the final gene trees used in downstream analyses.

The two genes causing flower colour divergence in sympatric $P$. drummondii populations due to reinforcement (Flavonoid 3'5' hydroxylase and R2R3-Myb transcription factor, Hopkins \& Rausher 2011) were not recovered in all species and therefore were not included in any of the analyses.

\section{Genomewide differences between the species}

SNP detection. We used BWA ( $\mathrm{Li} \&$ Durbin 2009) to align trimmed Illumina reads from each of the five samples to the ortholog consensus sequences we created as described above. We combined alignments from the different samples using samtools (Li et al. 2009) and used the POPOOLATION2 PIPELINE (Kofler et al. 2011) to identify variable positions. This provided us with the number of reads for each allele in the five samples. For each sample, we called a heterozygous site if the allele with less coverage represented at least $10 \%$ of the reads. These alignments were used for the G-PHOcs analysis described below.

Diversity and divergence. We used sequence alignments from orthology inference (without heterozygous positions) to calculate sequence similarity using the $D$ statistic (Nei \& Li 1979) for all pairwise comparisons of Phlox species (Table S4, Supporting information), where $D$ is defined as number of diverged sites between two species divided by the alignment length. We then calculated the relative node depth (RND) of two taxa compared to an outgroup (Feder et al. 2005). RND is calculated as the divergence between two species (as measured by $d$ ) divided by the average divergence between each species and the outgroup (in our case, $P$. pilosa, also as measured by $d$ ).

\section{Species tree estimation}

The Phlox species tree was obtained using four different methods: (i) maximum-likelihood estimation with RAXML (Stamatakis 2014), (ii) majority rule extended consensus from the set of gene trees using PHYLIP (Felsenstein 2002), (iii) pseudolikelihood coalescent-based species tree estimation using MP-EST (Liu et al. 2010) and (iv) maximum parsimony using PHYLONET (Than et al. 2008). Methods (i) and (iv) employed an alignment that was created by concatenating ortholog alignments at least $400 \mathrm{bp}$ long that included the full taxon set (all five samples) and no gaps. All methods converged on the same species tree (Table S3, Supporting information).

\section{Analyses of introgression}

$D$-statistic. We tested for introgression between Texas Phlox species using the D-statistic, otherwise known as the ABBA-BABA test, which compares counts of discordant site patterns (Durand et al. 2011). For this analysis, we used ortholog alignments that contained all five samples were at least $400 \mathrm{bp}$ long and had no gaps; 131 orthologs were discarded from this analysis because they were monomorphic or too short. We had a total of 1978 orthologs consisting of 1111392 aligned base pairs and 42478 variable sites.

We used Hybridcheck (Ward \& Oosterhout 2015) to count the number of ABBA and BABA site patterns in two types of four-taxa phylogenies. First, we used phylogenies containing the four species with a single P. drummondii sample. We tested for introgression between P.drummondii and P. cuspidata, and between $P$. roemeriana and $P$. cuspidata using these trees. We counted sites for which the derived allele was present in both P. drummondii and P. cuspidata, but not in P. roemeriana (ABBA sites), and the number of variable sites for which the derived allele was present in P. cuspidata and P. roemeriana, but not in P.drummondii (BABA sites). We expect equal counts of the two site patterns (ABBA and BABA) when incomplete lineage sorting (ILS) causes discordance. If discordance is caused by introgression, we expect one of the site patterns to be more prevalent than the other. We performed these analyses using the dark-red $P$. drummondii sample (i.e. using the topology Roem, DruR, Cusp, Pilo) and the light-blue $P$. drummondii sample (i.e. using the topology Roem, DruB, Cusp, Pilo) separately.

Second, we counted discordant variable sites using a phylogeny containing the two P.drummondii samples, 
P. cuspidata and P. pilosa (i.e. DruB, DruR, Cusp, Pilo). This analysis allows testing for differential gene flow between either of the two P. drummondii samples and P. cuspidata.

We tested for differences in discordant site pattern counts using the D-statistic as implemented in Hybridcheck (Ward \& Oosterhout 2015). Although a full genome alignment (or other linkage information) is not available for Phlox, the transcriptome loci we sampled likely represent a random sample of mostly unlinked markers from across the genome. Under these circumstances, we used a jackknife approach to test for genomewide variation in incongruence (Meyer et al. 2012; Eaton \& Ree 2013).

Phylogenetic inference of introgression. If there is introgression among Texas annual Phlox species, their relationship can be inferred using a phylogenetic network that allows for reticulate events (Huson \& Bryant 2006; Huson et al. 2011). We created a phylogenetic network using the PHYLONETWORKS software (Solís-Lemus \& Ané 2016) and all 2109 gene trees that contained all five samples (the two P.drummondii and the three other Phlox species), with P. pilosa as an outgroup. We used SNAQ (Solís-Lemus \& Ané 2016) to evaluate the most likely network given the species tree and gene trees and to calculate $\gamma$, the vector of inheritance probabilities, describing the proportion of genes inherited by a hybrid node from one of its parents.

We also used a Bayesian coalescent-based approach to estimate introgression rates and divergence times in our Phlox phylogeny as implemented in G-PHocs (Gronau et al. 2011). G-PHOCS conditions its inference on a given phylogeny and uses information from local genealogies at short loci to generate estimates of demographic parameters (Gronau et al. 2011). This method integrates over possible phasings of diploid genotypes, making use of both chromosomes per individual. We used custom Perl scripts to create diploid sequence alignments for orthologs using genotypes from read mapping. IUPAC ambiguity codes (R, Y, S, W, M and K) were used for heterozygous sites. We included 2937 orthologs from our five sampled taxa in the analysis.

To test for introgression between Phlox species in GPHOCs, we created 14 'migration bands' representing all possible directions of gene flow between branches of our 5-taxon phylogeny (Table S5, Supporting information). We computed the posterior mean and the $95 \%$ Bayesian credible interval for divergence times $(\tau)$, population sizes $(\theta)$ and migration rates $(m)$ in mutation units (Table S5, Supporting information). To obtain estimates of effective divergence times in years, we assumed a generation time of 1 year and used the average substitution rate for land plants of $\mu=4 \times 10^{-9}$
(Magallón et al. 2013). The divergence time equals: $T_{\text {div }}=(\tau+0.5 \times \theta) / \mu$. To obtain the per generation migration rate $\left(M_{\text {gen }}\right.$, the proportion of individuals that arose by migration per generation), we multiplied $m$ by the mutation rate: $M_{\text {gen }}=m \times \mu$. Finally, to obtain the total migration rate $\left(M_{\text {total }}\right)$, we multiplied the per generation rate times the number of generations that migration is allowed: $M_{\text {total }}=M_{\text {gen }} \times T_{\text {div }} . M_{\text {total }}$ represents the probability that a lineage from the target population originated in the source population and is equivalent to $\gamma$ from SNAQ.

Testing for recent gene flow between P. drummondii and P. cuspidata. As discussed in the 'Results', we find evidence of gene flow between P. cuspidata and the darkred $P$. drummondii sample, but not between $P$. cuspidata and the light-blue P.drummondii sample. We hypothesize this inconsistency is due to recent gene flow in sympatry, as represented by the dark-red sample, and a lack of recent gene flow in allopatry, as represented by the light-blue sample. To test this hypothesis, we investigated patterns of relative divergence in genes for which the $P$.drummondii sequences were sister to $P$. cuspidata sequences.

Although both the light-blue and the dark-red $P$. drummondii samples have many genes showing sister relationships with $P$.cuspidata, we hypothesize the excess of genes with this discordant gene tree in the dark-red sample is due to gene flow in sympatry. Both ILS and introgression can result in the same gene tree topology, but relative divergence between sequences in different taxa showing introgression is predicted to be much less than divergence of sequences that underwent ILS (Fig. 4a). We therefore predict that if the excess of genes showing discordant gene trees in the dark-red sample is due to gene flow, these genes will have a lower relative divergence with $P$. cuspidata sequences than genes that show the same gene tree topology in the light-blue sample. To test this prediction, we used the set of orthologs containing all five samples (a total of 2109 genes) to create two sets of four-taxon gene trees, one using the dark-red $P$. drummondii sample, the other the using the light-blue $P$. drummondii sample. For each of these two sets of gene trees, we identified all the discordant trees for which $P$. drummondii and P. Cuspidata were sister taxa (they are not sister in the inferred species tree). We then classified these genes into three categories: genes that show this topology (i) for both P. drummondii samples ('Both', 433 genes), (ii) for just the dark-red P.drummondii sample ('DruR only', 193 genes) and (iii) for just the light-blue P.drummondii sample ('DruB only', 63 genes).

If gene flow is recent, we expect the 'DruR only' category to be enriched with recently introgressed loci, 
having relatively low divergence between $P$. drummondii and P. cuspidata. We predict that the 'DruB only' and 'Both' data sets will consist mostly of ancestral polymorphism sampled in both lineages due to ILS. We measured divergence between the dark-red P. drummondii and $P$. cuspidata lineages (i.e. $\mathrm{RND}_{\text {DruB-Cusp), and compared }}$ these values between genes contained in the 'DruR only' and 'Both' categories. Similarly, we compared the genes in the 'DruB only' and 'Both' categories using the divergence between the light-blue $P$. drummondii and $P$. cuspi-

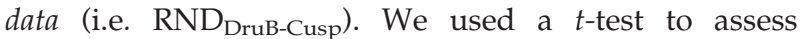
significant differences between categories. Our expectation is that the genes showing $P$. drummondii sister to $P$. cuspidata in only the dark-red sample are recently introgressed and will therefore have a lower RND than the genes showing the same topology in both P.drummondii samples, for which discordance should be likely due to ILS. The same pattern of lower RND is not expected in the light-blue only category, as recent introgression is not predicted in the recent history of this allopatric individual. Because our gene tree categories contained different numbers of genes, in addition to testing for RND differences between categories, we calculated $95 \%$ bootstrap confidence intervals.

Similar to an approach used to identify gene flow in humans (Kuhlwilm et al. 2016) and other apes (de Manuel et al. 2016), we also compared the genetic divergence between $P$. drummondii and P. cuspidata with the divergence between $P$. drummondii samples. For each of the ortholog groups containing all five samples, we calculated three values of genetic divergence: $R \mathrm{ND}_{\text {DruR-Cusp }}$ $\mathrm{RND}_{\text {DruB-Cusp }}$ and $\mathrm{RND}_{\text {DruB-DruR. We created two sets }}$ containing the genes with lowest values of interspecific divergence using the lower $5 \%$ tails of the distribution of $\mathrm{RND}_{\text {DruB-Cusp }}$ and RND $\mathrm{DruR}_{\text {-Cusp. Following similar logic }}$ outlined above, candidates for recent introgression will likely be in these 5\% tails. We compared the mean intraspecific divergence (i.e. $\mathrm{RND}_{\text {DruB-DruR }}$ ) between these two sets using a $t$-test. Our expectation is that genes showing low divergence between $P$.drummondii and $P$. cuspidata because of recent gene flow will show high divergence between the two $P$.drummondii samples. Confidence intervals for each category were determined using bootstrap resampling with replacement within each category. This analysis was conducted in $\mathrm{R}$.

\section{Results}

\section{Transcriptome assembly and orthology inference}

We carried out de novo transcriptome assembly from multiple species and identified orthologs in these assemblies to study the evolutionary history of a small clade of Phlox wildflowers native to Texas. Our assembled transcriptomes contained between 73000 and 105000 putative genes with $1.5 \pm 0.2$ 'isoforms' (i.e. putative haplotypes or splice variants) per gene (Table 1). We detected open reading frames (ORFs) in $36-47 \%$ of the transcripts, but only $20 \%$ of these ORFs contained both start and stop codons. Our short contig lengths (mean $\mathrm{N} 50=582 \pm 174$ ) indicate that a large proportion of genes were not entirely assembled. For orthology inference and phylogenetic reconstruction, we only used CDS and excluded UTRs and noncoding transcripts, as this facilitates sequence alignment and minimizes the amount of missing sequence resulting from RNA degradation or sequencing errors (Yang \& Smith 2014). Using the MI method of orthology inference (Dunn et al. 2008, 2013; Smith et al. 2011), we identified more than 2000 ortholog clusters containing all five samples. Orthologous regions from different species were $97 \%$ identical on average, while orthologs from the two Phlox drummondii samples shared $98 \%$ identity.

\section{Phylogenetic relationship between Texas annual Phlox}

We inferred the phylogenetic relationships among Texas Phlox species using maximum likelihood and maximum parsimony with concatenated sequence alignments, and consensus and coalescent-based methods with individual gene trees. All of our analyses placed $P$. drummondii sister to Phlox roemeriana (Figs $1 \mathrm{~b}$ and S2, Table S3, Supporting information). These relationships had 100\% bootstrap support in analyses of concatenated sequences, but were less supported in consensus analyses (incongruence is reflected in concordance factors, which ranged from $36 \%$ to 55\%; see Fig. 1 and Table S3, Supporting information).

\section{Evidence for introgression between P. cuspidata and P. drummondii}

D-statistic. Nearly $60 \%$ of individual gene trees were inconsistent with the species tree (Fig. 2a, b). Our fourspecies (including the outgroup) phylogeny allows for two possible discordant gene trees: one containing the clade (Phlox cuspidata, P.drummondii), the other with (P. cuspidata, P. roemeriana). Using the dark-red P. drummondii sample, more genes were observed to support P. drummondii and P. cuspidata as sister species (35\% of all gene trees) than P. roemeriana and P. cuspidata (25\% of all gene trees). This asymmetry in the proportion of discordant phylogenies suggests gene flow between P. drummondii and P. cuspidata. No such asymmetry was detected using the light-blue $P$. drummondii sample (Fig. 2b).

We explicitly tested for asymmetry in discordance patterns using the D-statistic (Green et al. 2010; Durand 

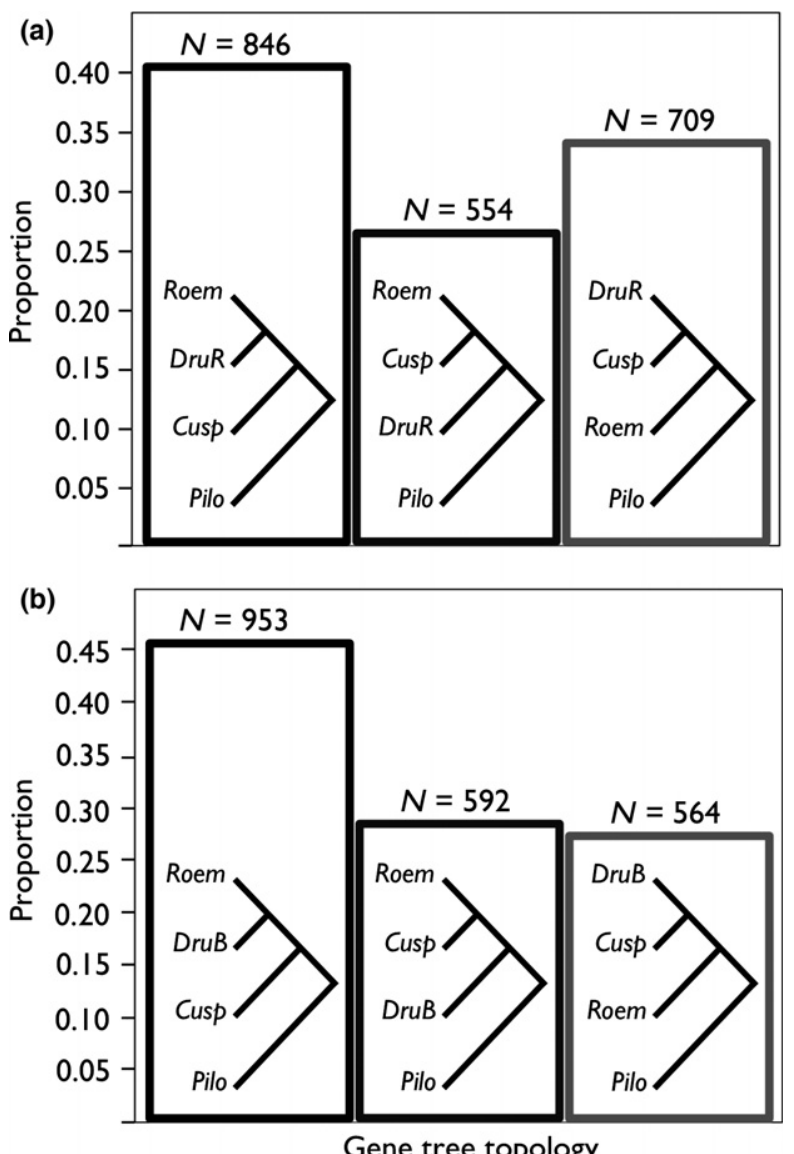

Fig. 2 Gene tree patterns. Number and proportion of gene trees presenting the three possible four-species topologies with the dark-red Phlox drummondii sample (a) and the light-blue $P$. drummondii sample (b). DruR = dark-red P. drummondii; DruB $=$ light-blue $\quad P$. drummondii $; \quad$ Roem $=$ Phlox roemeriana $;$ Cusp $=$ Phlox cuspidata $;$ Pilo $=$ Phlox pilosa .

et al. 2011). Because this test requires sets of four taxa, we conducted three tests: to test for introgression between $P$. cuspidata and $P$.drummondii or between $P$. cuspidata and $P$. roemeriana we calculated $D$ using a phylogeny with only one of the two P. drummondii samples and the three other species. We observed a significantly positive $D$ value for analyses including the darkred sample $\left(N_{\mathrm{ABBA}}=1787, N_{\mathrm{BABA}}=1282 ; D=0.165\right.$, $P<0.001$ ), which suggests gene flow between $P$. cuspidata and dark-red P.drummondii. We did not find strong support for gene flow in analyses including the light-blue $P$. drummondii and the other three species $\left(N_{\text {ABBA }}=1145, \quad N_{\text {BABA }}=1391 ; D=-0.097, \quad P=0.031\right)$. Note that $D$ in this analysis is negative, suggesting gene flow between $P$. roemeriana and $P$. cuspidata. To directly compare gene flow between P. cuspidata and the two $P$. drummondii samples, we performed this test using a phylogeny containing both the light-blue and the darkred P. drummondii, P. cuspidata and P. pilosa. We found a positive and highly significant $D$ value $\left(N_{\mathrm{ABBA}}=1323\right.$, $\left.N_{\mathrm{BABA}}=572 ; D=0.396, P<0.001\right)$, indicating that the dark-red $P$. drummondii sample has exchanged genes with $P$. cuspidata. It is important to note that the $D$-statistic suggests the presence of introgression but cannot be used to determine absolute rates of gene flow. We therefore used multiple additional phylogenetic methods to determine the most likely migration events between Phlox samples.

Phylogenetic inference using networks. We used a recently developed method (Solís-Lemus \& Ané 2016) to infer a phylogenetic network of the Texas Phlox samples from individual gene trees (Fig. 3). Unlike in the ABBABABA tests, we used all five samples for this analysis. Consistent with our previous results, the phylogenetic network that best fits our data included one hybrid edge connecting the branches leading to the dark-red P. drummondii and P. cuspidata. The direction of this hybrid node, which reflects the direction of gene flow, was from P.cuspidata into the sympatric dark-red $P$. drummondii. The vector of inheritance probabilities $(\gamma)$ for this node was estimated to be 0.25 (Solís-Lemus \& Ané 2016). We also used a Bayesian approach (Gronau et al. 2011) to infer migration rates among all branches in our phylogenetic tree. The inferred demographic model suggests gene flow from $P$. cuspidata into the dark-red P.drummondii (Fig. 3). We also found

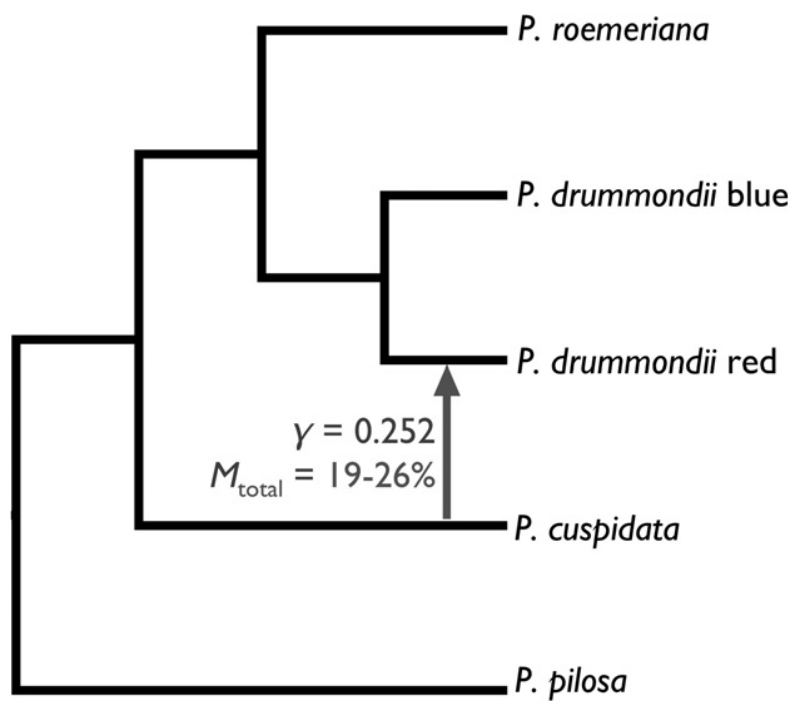

Fig. 3 Introgression model of Phlox history. Phylogeny of Texas Phlox with the migration event inferred by both G-PHOCS and SNAQ represented as a grey arrow. Next to the arrow are the probability of hybrid descent $(\gamma)$ estimated with SNAQ and the 95\% Bayesian credible intervals of total migration rates $\left(M_{\text {total }}\right)$ estimated with G-PHOcs. We also inferred four minor gene flow events $(<1 \%$ total migration rate, Table S5, Supporting information) with G-PHOcs. 
(a) ILS

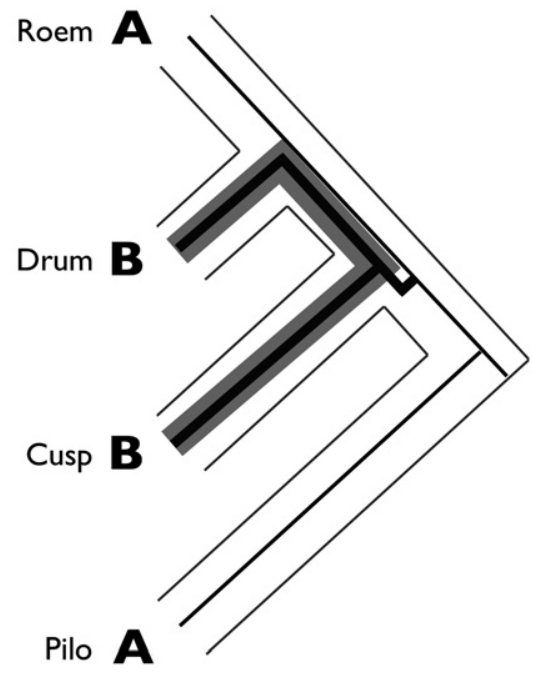

Introgression

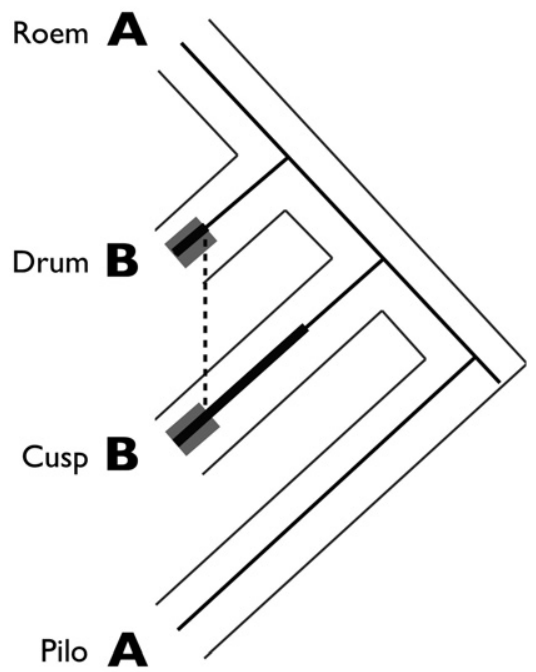

(b)

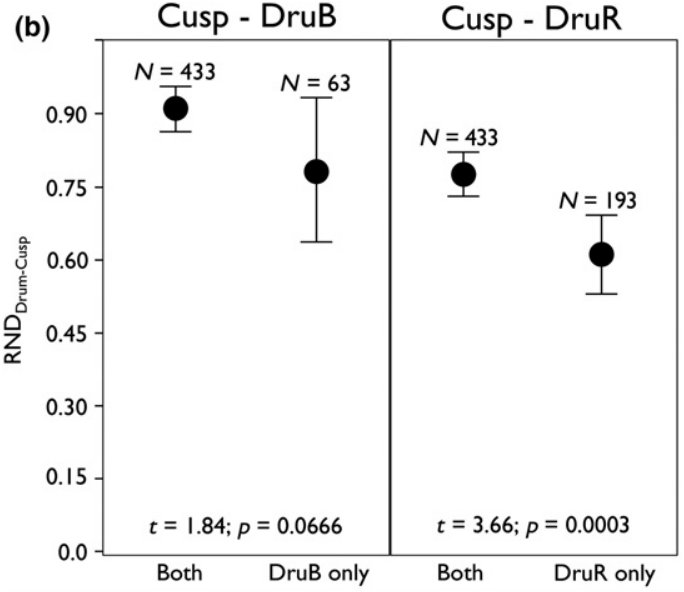

(c)

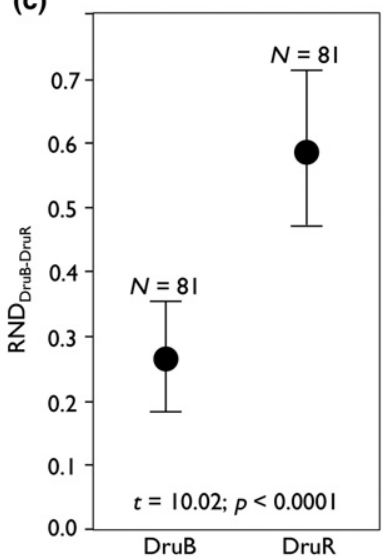

Fig. 4 Tests of recent introgression in sympatry. (a) Schematic showing how introgression and incomplete lineage sorting create discordant phylogenetic signals. We represent a derived allele (' $\mathrm{B}$ '-thick line) that is shared between Phlox drummondii and Phlox cuspidata. We use grey highlighting to indicate areas on the gene trees for which the derived allele is independently diverging in the two taxa. Introgressed loci are expected to show lower interspecific divergence than loci showing similarity due to incomplete lineage sorting. (b) Interspecific divergence for genes that show P. drummondii as sister to P. cuspidata in both $P$. drummondii samples ('Both'), only in the light-blue $P$. drummondii sample ('DruB only') and only in the dark-red P. drummondii sample ('DruR only'). RND was calculated either comparing $P$. cuspidata and the light-blue P. drummondii ('Cusp-DruB') or P. cuspidata and the dark-red P. drummondii ('CuspDruB'$^{\prime}$ ). (c) Intraspecific divergence in genes showing low interspecific divergence in the light-blue $P$. drummondii (DruR) and dark-red P. drummondii (DruR). Introgressed loci are expected to show higher intraspecific divergence. Results from $t$-tests comparing the means are shown. Bars indicate 95\% confidence intervals as calculated using bootstrap resampling. some support for very low levels of gene flow from the light-blue $P$.drummondii into $P$. roemeriana, from the ancestors of P.drummondii into P. cuspidata, and between $P$. roemeriana and P. cuspidata (Fig. 3, Table S5, Supporting information).

Using levels of divergence to test for gene flow. The results from both the $D$-statistic and the phylogenetic analyses indicate gene flow between P. cuspidata and the darkred $P$. drummondii, but not between $P$. cuspidata and the light-blue $P$. drummondii. We further tested the hypothesis that this difference is due to recent introgression between the sympatric species using two methods. First, we investigated the divergence in genes with topologies showing P. drummondii sister to P. cuspidata. This method compares the genetic divergence between genes with discordant topologies to distinguish between ILS and introgression (see 'Methods' and Fig. 4a, b). We predict that recently introgressed loci will have discordant gene tree topologies and display low interspecific divergence. We found that genes that have P. drummondii sister to P. cuspidata in only the dark-red sample had a significantly lower RND than genes where $P$. cuspidata is sister to both $P$. drummondii samples (Fig. $4 \mathrm{~b}$, right side). Genes showing P. drummondii sister to $P$. cuspidata only in light-blue samples have RND values indistinguishable from genes showing this topology in both samples (Fig. 4b, left side). These findings support our hypothesis that the dark-red P. drummondii experienced recent introgression from P. cuspidata.

Second, we compared the genetic divergence between P. drummondii and P. cuspidata to that between the two P. drummondii samples (as in Kuhlwilm et al. 2016). We found that genes in the dark-red $P$. drummondii genome that show low divergence with $P$. cuspidata also have high divergence in comparison with the light-blue P. drummondii genome (Fig. 4c). In contrast, light-blue 
P. drummondii genes that show low divergence with $P$. cuspidata show much less divergence with dark-red P. drummondii (Fig. 4c). These patterns support the hypothesis of recent gene flow in sympatry.

\section{Discussion}

Hybridization is a pervasive evolutionary force that can influence the process of speciation. Can hybridization and gene flow prevent the evolution of reproductive isolation in sympatry? New genomic analyses provide an exciting opportunity to test theories on the impact of hybridization during lineage diversification (Gompert \& Buerkle 2016; Payseur \& Rieseberg 2016; Vallejo-Marín $\&$ Hiscock 2016). Here we use transcriptome sequencing to ask whether gene flow occurred during a classic example of reinforcement in Texas Phlox. Using multiple analyses of phylogenetic discordance, we demonstrate that recent gene flow occurred between sympatric Phlox drummondii and Phlox cuspidata.

\section{Phylogenetic relationships between annual Phlox}

Previous studies across the Phlox genus indicate that the three species P. drummondii, P. cuspidata and Phlox roemeriana form a monophyletic clade (Ferguson et al. 1999a; Ferguson \& Jansen 2002). These are the only annual species in the genus, and they have overlapping ranges in Texas. According to our transcriptome-wide phylogenetic analysis, P. roemeriana and P. drummondii are sister species (Fig. 1b). These relationships were supported by both P. drummondii samples (Fig. 2) but were unexpected given that $P$. roemeriana has multiple derived traits, including five ovules per locule instead of one, a long calyx, and a yellow eyespot on the corolla (Ferguson et al. 1999a). Incongruence between individual gene trees indicates ILS and possibly gene flow during the divergence of these species. Given the abundance of discordant gene trees, it is not surprising that previous phylogenetic analyses based on limited gene sampling suggested that $P$. roemeriana was sister to P. cuspidata (Ferguson et al. 1999a; Ferguson \& Jansen 2002).

\section{The impact of gene flow during reinforcement}

Four-taxon analyses of introgression provided strong evidence for gene flow between the sympatric dark-red P. drummondii and P. cuspidata. Furthermore, phylogenetic network analysis and a Bayesian phylogenetic approach also indicate that the most likely evolutionary history of these species includes gene flow from the $P$. cuspidata lineage into the dark-red $P$. drummondii lineage (Fig. 3). We do not believe our results are due to sampling a rare backcross individuals as our estimates of gene flow between P. cuspidata and the dark-red $P$. drummondii are too low for an early hybrid, and are comparable to levels found in species with moderate levels of hybridization (Cahill et al. 2016; Solís-Lemus \& Ané 2016). Together, these analyses demonstrate that reinforcement in sympatry between $P$. drummondii and $P$. cuspidata occurred despite gene flow between these taxa. This is one of the first empirical demonstrations that reinforcement does occur in nature in the presence of gene flow.

Hybrids between P.drummondii and P. cuspidata are observed in the field (Levin 1967, 1975, 1985; Ferguson et al. 1999b; Ruane \& Donohue 2008; Ruane 2009) but have high sterility (Ruane \& Donohue 2008). Commongarden field experiments indicate that these hybridization events are twice as frequent when $P$.drummondii has the ancestral light-blue flower colour than when it has the derived dark-red flower colour (Hopkins et al. 2012), suggesting that prior to flower colour divergence hybridization was more frequent. This high hybridization rate not only provides the source of selection driving reinforcement, but also allows for gene flow between these sympatric species. Here, we confirm that the production of these hybrids resulted in gene flow between P. drummondii and P. cuspidata. Although we found no evidence of greater gene flow before divergence of the allopatric and sympatric P.drummondii populations (Fig. 3), the fast evolution of flower colour under strong reinforcing selection would limit our ability to distinguish between gene flow that occurred during and after flower colour divergence.

How did reinforcement successfully cause flower colour divergence in $P$. drummondii despite gene flow? In order for new alleles causing assortative mating between sympatric species to evolve, they must remain in linkage disequilibrium with alleles causing hybrid sterility or inviability (Servedio 2009). Population genetic theory predicts that selection can build up linkage disequilibria between uninked loci (Kimura 1956; Slatkin 1975). Furthermore, theoretical work specifically demonstrates that strong selection can drive the evolution of RI even when diverging lineages experience gene flow (Liou \& Price 1994; Caisse \& Antonovics 1978; Dickinson \& Antonovics 1973; Servedio \& Kirkpatrick 1997). Therefore, the strength of reinforcing selection likely enabled the successful evolution of flower colour divergence in $P$. drummondii despite gene flow with $P$. cuspidata.

The strength of reinforcing selection is determined by the amount of hybridization and the cost of hybridization. In Phlox, the hybridization rate between light-blue P. drummondii and P. cuspidata is high, with commongarden estimates at $40 \%$ (Hopkins et al. 2012), and the 
cost of hybridization is high, with sterility estimated between $50 \%$ and $100 \%$ (Ruane \& Donohue 2008). Previous research determined that flower colour divergence between allopatric and sympatric populations is caused by strong selection; selection coefficients are estimated to be 0.14 at one locus and 0.47 at the other flower colour locus (Hopkins et al. 2014). This selection is likely strong enough to explain how reinforcement successfully evolved in sympatric Phlox populations despite gene flow.

Gene flow can prevent the evolution of reinforcement by allowing recombination to break down the genetic associations between alleles causing assortative mating and alleles causing post-zygotic or post-mating reproductive isolation. There are two hypothesized mechanisms to overcome the problem of recombination-oneallele mechanisms and reduced recombination mechanisms (Servedio 2009). The one-allele mechanism describes a scenario in which a novel allele causes assortative mating when present in either sympatric species (Felsenstein 1981). Flower colour variation in Phlox is not determined by one allele and therefore this mechanism cannot explain how reinforcement overcame gene flow in this system.

In the case of flower colour in Phlox, recombination could cause individuals with $P$. cuspidata alleles at the hybrid sterility loci to have dark-red flowers. This breakdown in the association between sterility alleles and flower colour alleles means assortative mating based on flower colour could result in sterile hybrids. Physical linkage that reduces recombination between alleles causing assortative mating and alleles causing hybrid sterility would increase the feasibility of reinforcement (Servedio 2009; Trickett \& Butlin 1994; Kirkpatrick \& Servedio 1999; ). For example, in the Ficedula flycatcher system, loci controlling divergence in plumage colour, which evolved due to reinforcement (Sætre et al. 2003), and loci associated with hybrid female sterility colocalize on the sex chromosomes (Sætre et al. 1997; Sæther et al. 2007). In Phlox, we do not know the genomic location of loci causing hybrid sterility or flower colour variation. Future research is necessary to determine whether genetic linkage, along with strong selection, can explain how increased assortative mating evolved despite gene flow.

\section{Asymmetric gene flow and asymmetric divergence}

Our phylogenetic analyses suggest that the most likely evolutionary history of these lineages involved asymmetric gene flow from $P$. cuspidata into $P$. drummondii. This presumably occurred because hybrids backcrossed with P.drummondii more than with P. cuspidata. This direction of gene flow agrees with predictions based on mating systems of these two species. P. cuspidata is self-compatible and undergoes high rates of self-fertilization, while P.drummondii is predominantly selfincompatible, and thus depends on out-crossed pollen for seed-set. Because P. cuspidata has a lower out-crossing rate than $P$.drummondii, there is less opportunity for receiving pollen from hybrid individuals, which could explain the asymmetric gene flow.

The asymmetric gene flow in Phlox is also consistent with the asymmetric morphological divergence in the sympatric zone: while $P$. drummondii displays a flower colour change in sympatry, P. cuspidata has the same flower morphology across its range. In all known cases of reinforcement in plants, only one of the two species diverges in phenotype in sympatry (Hopkins 2013). Why is divergence asymmetric? One leading hypothesis is that there is an asymmetric cost to hybridization causing reinforcing selection to be stronger in one species than the other (Jaenike et al. 2006; Cooley 2007). Our results support this hypothesis. More introgression of heterospecific genetic material into $P$. drummondii than into P. cuspidata could represent a higher cost to hybridization and explain why reinforcing selection caused divergence in one species and not the other. The predominant direction of gene flow should be confirmed with further sequencing of more samples as limited conclusions can be made from two individuals.

\section{Geographic variation in gene flow}

We performed genomic analyses of introgression using light-blue and dark-red flowered $P$. drummondii individuals. These plants are found in allopatry and sympatry with $P$. cuspidata, respectively. We found significant evidence for introgression between the dark-red sample and P. cuspidata, but not between P. cuspidata and the light-blue sample. This finding is consistent with gene flow between Phlox in sympatric populations.

We took advantage of the sister relationships between the two colour morphs in P. drummondii and their geographic separation to further test the hypothesis of introgression. We first compared divergence in genes with discordant phylogenetic signals. This analysis is based on two assumptions. First, recent gene flow is likely to result in geographic variation in introgression because, compared to ILS and ancient introgression, there is less time for novel alleles to spread across populations. Second, loci that were recently exchanged between species will show higher sequence similarity than loci undergoing ILS as introgressed sequences have had less evolutionary time to diverge in each lineage (Fig. 4a). Therefore, we predict that (i) sequences from sympatric samples will have more discordant gene tree topologies putting P. cuspidata and P.drummondii 
as sister taxa, and (ii) those sequences showing discordance only in sympatry will have lower between-species divergence than genes showing the same pattern of discordance in both allopatric and sympatric samples. Consistent with these predictions, we find an excess of genes supporting $P$. cuspidata as sister to $P$. drummondii using the dark-red sample, but not using the light-blue sample (Figs 2 and 4c). Furthermore, we find that those genes having the aforementioned discordant gene tree exclusively in the dark-red sample are significantly less diverged than those whose discordant gene trees were present in both samples (Fig. $4 \mathrm{~b}$ ).

We then compared interspecific genetic divergence to intraspecific divergence (Kuhlwilm et al. 2016). As expected under a scenario of recent introgression in sympatry, genes showing low divergence between the dark-red P. drummondii and P. cuspidata show high divergence between the two $P$.drummondii samples (Fig. 4c).

As with many genomewide analyses inferring gene flow, our study depends on analysing patterns across many loci in a limited number of individuals. Sequencing more individuals from throughout the Phlox ranges will provide stronger estimates of the timing and amount of gene flow across the landscape.

\section{Conclusions}

Our transcriptome analyses provide strong evidence of gene flow during the process of reinforcement. Introgression can be detected with a variety of methods (Payseur \& Rieseberg 2016), but unfortunately most of them cannot be applied to our data given the small number of samples and the use of transcriptomes composed of short contigs. Although all the analyses applied in this study were originally designed for a small number of samples (Green et al. 2010; Gronau et al. 2011; Payseur \& Rieseberg 2016), we acknowledge that some of our results will benefit from confirmation using more samples. In particular, sampling allopatric and sympatric individuals from the three species will allow a better quantification of the timing, magnitude and direction of gene flow. Larger genomic surveys will also allow us to explore the identity and function of genes exchanged between Phlox species.

This is a promising system for future analyses of introgression because of the extensive knowledge about the biogeography of the species (Erbe \& Turner 1962; Levin 1967; Ruane 2009) and the strength of reinforcing selection (Hopkins \& Rausher 2012, 2014; Hopkins et al. 2014). Future studies will be able to incorporate this biologically relevant information into studies of genomic variation between and within species.

\section{Acknowledgements}

We are very thankful for the advice on bioinformatic analyses provided by Rubén Sancho, Ben J. Ward, Rosa Fernández, Ya Yang, Claudia Solís-Lemus, Cécile Ané and the staff from FAS Research Computing. We also thank Dave Des Marais for his useful comments on our results.

\section{References}

Albert A, Schluter D (2004) Reproductive character displacement of male stickleback mate preference: reinforcement or direct selection? Evolution, 58, 1099-1107.

Altschul SF, Madden TL, Schäffer AA et al. (1997) Gapped BLAST and PSI-BLAST: a new generation of protein database search programs. Nucleic Acids Research, 25, 3389-3402.

Bank C, Hermisson J, Kirkpatrick M (2012) Can reinforcement complete speciation? Evolution, 66, 229-239.

Barton NH, De Cara MAR (2009) The evolution of strong reproductive isolation. Evolution, 63, 1171-1190.

Bolger AM, Lohse M, Usadel B (2014) Trimmomatic: a flexible trimmer for Illumina sequence data. Bioinformatics, 2, 8-13.

Butlin R (1987) Speciation by reinforcement. Trends in Ecology E Evolution, 2, 8-13.

Cahill JA, Fan Z, Gronau I et al. (2016) Whole-genome sequence analysis shows that two endemic species of North American wolf are admixtures of the coyote and gray wolf Science Advances, 2, e1501714.

Caisse M, Antonovics J (1978) Evolution in closely adjacent plant populations. Heredity, 40, 371-384.

Cooley JR (2007) Decoding asymmetries in reproductive character displacement. Proceedings of the Academy of Natural Sciences of Philadelphia, 156, 89-96.

Dickinson H, Antonovics J (1973) Theoretical considerations of sympatric divergence. The American Naturalist, 107, 256-274

Dunn CW, Hejnol A, Matus DQ et al. (2008) Broad phylogenomic sampling improves resolution of the animal tree of life. Nature, 452, 745-749.

Dunn CW, Howison M, Zapata F (2013) Agalma: an automated phylogenomics workflow. BMC Bioinformatics, 14, 330.

Durand EY, Patterson N, Reich D, Slatkin M (2011) Testing for ancient admixture between closely related populations. Molecular Biology and Evolution, 28, 2239-2252.

Eaton DA, Ree RH (2013) Inferring phylogeny and introgression using RADseq data: an example from flowering plants (Pedicularis: Orobanchaceae). Systematic Biology, 62, 689-706.

Enright AJ, Van Dongen S, Ouzounis CA (2002) An efficient algorithm for large-scale detection of protein families. Nucleic Acids Research, 30, 1575-1584.

Erbe L, Turner B (1962) A biosystematic study of the Phlox cuspidata-Phlox drummondii complex. American Midland Naturalist, 67, 257-281.

Feder JL, Xie X, Rull J et al. (2005) Mayr, Dobzhansky, and Bush and the complexities of sympatric speciation in Rhagoletis. Proceedings of the National Academy of Sciences of the United States of America, 102, 6573-6580.

Felsenstein J (1981) Skepticism towards Santa Rosalia, or why are there so few kinds of animals? Evolution, 35, 124-138.

Felsenstein J (2002) PHYLIP (Phylogeny Inference Package) (Version 3.52). 1993 Distributed by the author. Department of 
Genome Sciences, University of Washington, Seattle, Washington.

Ferguson CJ, Jansen RK (2002) A chloroplast DNA phylogeny of eastern Phlox (Polemoniaceae): implications of congruence and incongruence with the ITS phylogeny. American Journal of Botany, 89, 1324-1335.

Ferguson CJ, Kramer F, Jansen RK (1999a) Relationships of eastern North American Phlox (Polemoniaceae) based on ITS sequence data. Systematic Botany, 24, 616-631.

Ferguson CJ, Levin DA, Jansen RK (1999b) Natural hybridization between an outcrossing and a selfing Phlox (Polemoniaceae): the maternal species of F1 hybrids. Plant Systematics and Evolution, 218, 153-158.

Gerhardt HC (1994) The evolution of vocalization in frogs and toads. Annual Review of Ecology and Systematics, 25, 293-324.

Gompert Z, Buerkle CA (2016) What, if anything, are hybrids: enduring truths and challenges associated with population structure and gene flow. Evolutionary Applications, 9, 909-923.

Grabherr MG, Haas BJ, Yassour M et al. (2011) Trinity: reconstructing a full-length transcriptome without a genome from RNA-Seq data. Nature Biotechnology, 29, 644.

Green RE, Krause J, Briggs AW et al. (2010) A draft sequence of the Neandertal genome. Science, 328, 710-722.

Gronau I, Hubisz MJ, Gulko B, Danko CG, Siepel A (2011) Bayesian inference of ancient human demography from individual genome sequences. Nature Genetics, 43, 1031-1034.

Haas BJ, Papanicolaou A, Yassour M et al. (2013) De novo transcript sequence reconstruction from RNA-seq using the Trinity platform for reference generation and analysis. Nature Protocols, 8, 1494-1512.

Höbel G, Gerhardt HC (2003) Reproductive character displacement in the acoustic communication system of green tree frogs (Hyla cinerea). Evolution, 57, 894-904.

Holder MT, Anderson JA, Holloway AK (2001) Difficulties in detecting hybridization. Systematic Biology, 50, 978-982.

Hopkins R (2013) Reinforcement in plants. New Phytologist, 197, 1095-1103.

Hopkins R, Rausher MD (2011) Identification of two genes causing reinforcement in the Texas wildflower Phlox drummondii. Nature, 469, 411-414.

Hopkins R, Rausher MD (2012) Pollinator-mediated selection on flower color allele drives reinforcement. Science, 335, 1090-1092.

Hopkins R, Rausher MD (2014) The cost of reinforcement: selection on flower color in allopatric populations of Phlox drummondii. The American Naturalist, 183, 693-710.

Hopkins R, Levin DA, Rausher MD (2012) Molecular signatures of selection on reproductive character displacement of flower color in Phlox drummondii. Evolution, 66, 469-485.

Hopkins R, Guerrero RF, Rausher MD, Kirkpatrick M (2014) Strong reinforcing selection in a Texas wildflower. Current Biology, 24, 1995-1999.

Hoskin CJ, Higgie M, McDonald KR, Moritz C (2005) Reinforcement drives rapid allopatric speciation. Nature, 437, 1353-1356.

Huson DH, Bryant D (2006) Application of phylogenetic networks in evolutionary studies. Molecular Biology and Evolution, 23, 254-267.

Huson DH, Rupp R, Scornavacca C (2011) Phylogenetic networks: concepts, algorithms and applications. Cambridge University Press, Cambridge.
Jaenike J, Dyer KA, Cornish C, Minhas MS (2006) Asymmetrical reinforcement and Wolbachia infection in Drosophila. PLoS Biology, 4, e325.

Joly S, McLenachan PA, Lockhart PJ (2009) A statistical approach for distinguishing hybridization and incomplete lineage sorting. The American Naturalist, 174, E54-E70.

Katoh K, Standley DM (2013) MAFFT multiple sequence alignment software version 7: improvements in performance and usability. Molecular Biology and Evolution, 30, 772-780.

Kimura M (1956) A model of a genetic system which leads to closer linkage by natural selection. Evolution, 10, 278287.

Kirkpatrick M (2000) Reinforcement and divergence under assortative mating. Proceedings of the Royal Society of London B: Biological Sciences, 267, 1649-1655.

Kirkpatrick M, Ravigne V (2002) Speciation by natural and sexual selection: models and experiments. The American Naturalist, 159, S22-S35.

Kirkpatrick M, Servedio MR (1999) The reinforcement of mating preferences on an island. Genetics, 151, 865-884.

Kofler R, Pandey RV, Schlötterer C (2011) PoPoolation2: identifying differentiation between populations using sequencing of pooled DNAsamples (Pool-Seq). Bioinformatics, 27, 3435-3436.

Kuhlwilm M, Gronau I, Hubisz MJ et al. (2016) Ancient gene flow from early modern humans into Eastern Neanderthals. Nature, 530, 429-433.

Kulathinal RJ, Stevison LS, Noor MA (2009) The genomics of speciation in Drosophila: diversity, divergence, and introgression estimated using low-coverage genome sequencing. PLoS Genetics, 5, e1000550.

Levin DA (1967) Hybridization between annual species of Phlox: population structure. American Journal of Botany, 54, 1122-1130.

Levin DA (1975) Interspecific hybridization, heterozygosity and gene exchange in Phlox. Evolution, 29, 37-51.

Levin DA (1985) Reproductive character displacement in Phlox. Evolution, 39, 1275-1281.

Li H, Durbin R (2009) Fast and accurate short read alignment with Burrows-Wheeler transform. Bioinformatics, 25, 1754-1760.

Li H, Handsaker B, Wysoker A et al. (2009) The sequence alignment/map format and SAMtools. Bioinformatics, 25, 2078-2079.

Li W, Godzik A (2006) Cd-hit: a fast program for clustering and comparing large sets of protein or nucleotide sequences. Bioinformatics, 22, 1658-1659.

Liou LW, Price TD (1994) Speciation by reinforcement of premating isolation. Evolution, 48, 1451-1459.

Liu L, Yu L, Edwards SV (2010) A maximum pseudo-likelihood approach for estimating species trees under the coalescent model. BMC Evolutionary Biology, 10, 302.

Magallón S, Hilu KW, Quandt D (2013) Land plant evolutionary timeline: gene effects are secondary to fossil constraints in relaxed clock estimation of age and substitution rates. American Journal of Botany, 100, 556-573.

Mallet J, Besansky N, Hahn MW (2016) How reticulated are species? BioEssays, 38, 140-149.

de Manuel M, Kuhlwilm M, Frandsen P et al. (2016) Chimpanzee genomic diversity reveals ancient admixture with bonobos. Science, 354, 477-481.

Martin SH, Davey JW, Jiggins CD (2015) Evaluating the use of ABBA-BABA statistics to locate introgressed loci. Molecular Biology and Evolution, 32, 244-257. 
Matute DR (2010) Reinforcement can overcome gene flow during speciation in Drosophila. Current Biology, 20, 2229-2233.

Meyer M, Kircher M, Gansauge M-T et al. (2012) A high-coverage genome sequence from an archaic Denisovan individual. Science, 338, 222-226.

Nei M, Li W-H (1979) Mathematical model for studying genetic variation in terms of restriction endonucleases. Proceedings of the National Academy of Sciences of the United States of America, 76, 5269-5273.

Noor MA (1995) Speciation driven by natural selection in Drosophila. Nature, 375, 674-675.

Nosil P (2013) Degree of sympatry affects reinforcement in Drosophila. Evolution, 67, 868-872.

Nosil P, Crespi B, Sandoval C (2003) Reproductive isolation driven by the combined effects of ecological adaptation and reinforcement. Proceedings of the Royal Society of London B: Biological Sciences, 270, 1911-1918.

Ortiz-Barrientos D, Counterman BA, Noor MAF (2004) The genetics of speciation by reinforcement. PLoS Biology, 2, 2256-2263.

Payseur BA, Rieseberg LH (2016) A genomic perspective on hybridization and speciation. Molecular Ecology, 25, 2337-2360.

Price MN, Dehal PS, Arkin AP (2010) FastTree 2-approximately maximum-likelihood trees for large alignments. PLoS One, 5, e9490.

Rosenzweig BK, Pease JB, Besansky NJ, Hahn MW (2016) Powerful methods for detecting introgressed regions from population genomic data. Molecular Ecology, 25, 2387-2397.

Ruane LG (2009) Mating system and the hybridization between self-compatible Phlox cuspidata and self-incompatible Phlox drummondii. Evolutionary Ecology, 23, 791-805.

Ruane LG, Donohue K (2008) Pollen competition and environmental effects on hybridization dynamics between Phlox drummondii and Phlox cuspidata. Evolutionary Ecology, 22, 229 241.

Sæther SA, Sætre G-P, Borge T et al. (2007) Sex chromosomelinked species recognition and evolution of reproductive isolation in flycatchers. Science, 318, 95-97.

Sætre G, Moum T, Bures S et al. (1997) A sexually selected character displacement in flycatchers reinforces premating isolation. Nature, 387, 589-592.

Sætre GP, Borge T, Lindroos K et al. (2003) Sex chromosome evolution and speciation in Ficedula flycatchers. Proceedings of the Royal Society of London B: Biological Sciences, 270, 53-59.

Sanderson N (1989) Can gene flow prevent reinforcement? Evolution, 43, 1223-1235.

Servedio MR (2009) The role of linkage disequilibrium in the evolution of premating isolation. Heredity, 102, 51-56.

Servedio MR, Kirkpatrick M (1997) The effects of gene flow on reinforcement. Evolution, 51, 1764-1772.

Servedio MR, Noor MA (2003) The role of reinforcement in speciation: theory and data. Annual Review of Ecology, Evolution, and Systematics, 34, 339-364.

Slatkin M (1975) Gene flow and selection in a two-locus system. Genetics, 81, 787-802.

Smadja C, Ganem G (2005) Asymmetrical reproductive character displacement in the house mouse. Journal of Evolutionary Biology, 18, 1485-1493.

Smadja CM, Loire E, Caminade P et al. (2015) Seeking signatures of reinforcement at the genetic level: a hitchhiking mapping and candidate gene approach in the house mouse. Molecular Ecology, 24, 4222-4237.

Smith SA, Dunn CW (2008) Phyutility: a phyloinformatics tool for trees, alignments and molecular data. Bioinformatics, 24, 715-716.

Smith SA, Wilson NG, Goetz FE et al. (2011) Resolving the evolutionary relationships of molluscs with phylogenomic tools. Nature, 480, 364-367.

Solís-Lemus C, Ané C (2016) Inferring phylogenetic networks with maximum pseudolikelihood under incomplete lineage sorting. PLoS Genetics, 12, e1005896.

Stamatakis A (2006) RAxML-VI-HPC: maximum likelihoodbased phylogenetic analyses with thousands of taxa and mixed models. Bioinformatics, 22, 2688-2690.

Stamatakis A (2014) RAxML version 8: a tool for phylogenetic analysis and post-analysis of large phylogenies. Bioinformatics, 30, 1312-1313.

Than C, Ruths D, Nakhleh L (2008) PhyloNet: a software package for analyzing and reconstructing reticulate evolutionary relationships. BMC Bioinformatics, 9, 322.

Trickett AJ, Butlin RK (1994) Recombination suppressors and the evolution of new species. Heredity, 73, 339-345.

Vallejo-Marín M, Hiscock SJ (2016) Hybridization and hybrid speciation under global change. New Phytologist, 211, 1170 1187.

Van Dongen S, Abreu-Goodger C (2012) Using MCL to extract clusters from networks. Bacterial Molecular Networks: Methods and Protocols, 804, 281-295.

Ward BJ, Oosterhout C (2015) HYBRIDCHECK: software for the rapid detection, visualization and dating of recombinant regions in genome sequence data. Molecular Ecology Resources, 16, 534-539.

Yang Y, Smith SA (2014) Orthology inference in nonmodel organisms using transcriptomes and low-coverage genomes: improving accuracy and matrix occupancy for phylogenomics. Molecular Biology and Evolution, 31, 3081-3092.

Yukilevich R (2012) Asymmetrical patterns of speciation uniquely support reinforcement in Drosophila. Evolution, 66, $1430-1446$.

F.R. and R.H. designed the experiments. F.R. and R.H. conducted laboratory and greenhouse work. F.R. and F.M. analysed data. F.R., F.M., M.H. and R.H. wrote the manuscript.

\section{Data accessibility}

Transcriptome Shotgun Assembly project has been deposited at DDBJ/EMBL/GenBank under accessions GFFR01000000 (Phlox drummondii Light blue), GFFP01 000000 (Phlox drummondii Dark red), GFFQ01000000 (Phlox roemeriana), GFFN01000000 (Phlox cuspidata), GFFO01000000 (Phlox pilosa). Gene trees, genotype calls and gene divergence data ( $d$ and RND) are deposited in Dryad (doi:10.5061/dryad.r25s5). 
2330 F. RODA ET AL.

\section{Supporting information}

Additional supporting information may be found in the online version of this article.

Table S1 Software and parameters used.

Table S2 Origin of samples used for transcriptome sequencing.
Table S3 Phylogenetic trees obtained with different methods.

Table S4 Genetic divergence $(d)$ and genetic relative divergence (RND) for all pairwise comparisons between annual Texas Phlox.

Table S5 Demographic parameters calculated with G-PHOcs. 